H. Umemura

Nagoya Math. J.

Vol. 47 (1972), 155-160

\title{
LA DIMENSION COHOMOLOGIQUE DES SURFACES ALGEBRIQUES
}

\author{
HIROSHI UMEMURA
}

En Géométrie Algébrique on a un critère pour qu'une surface moins une courbe soit affiine (Hartshorne (5)). Dans (5), on demande s'il existe un analogue analytique. Le but de cet article est de donner une condition numérique nécessaire pour les surfaces complexes compactes (Théorème 1) et une condition suffisante pour les surfaces réglées (Théorème 2).

Nous appelons une variété algébrique un schéma de type fini défini sur un corps algébriquement clos.

Je tiens à exprimer ma profonde gratitude à $M$. Hartshorne dont les conseils et les encouragements me furent une aide précieuse.

\section{Condition Necessaries.}

1.1. Dans ce paragraphe on donne une condition nécessaire pour qu'une surface moins une courbe soit une variété de Stein.

THÉORÈme 1. Soit $X$ une variété analytique complexe compacte de dimension 2, et soit $C$ une courbe irréductible non-singulière sur $X$. Si $X-C$ est une variété de Stein, alors $\left(C^{2}\right) \geq 0$ et $(C \cdot Y)>0$ pour toute courbe $Y$ sur $X$ différente de $C$.

Puisqu'un sous-ensemble analytique d'une variété de Stein est un espace de Stein, $X-D$ ne contient pas de courbe compacte, autrement dit $(C \cdot Y)$ est positif pour toute courbe $Y$ sur $X$ différente de $C$. Nous allons vérifier $\left(C^{2}\right) \geq 0$. Raisonnons par l'absurde. Si $\left(C^{2}\right)$ était négatif, d'après Grauert (4) il existerait une surface analytique complexe normale compacte $X^{\prime}$ et un morphisme $f$ de $X$ dans $X^{\prime}$ tels que l'image $f(C)$ de $C$ dans $X^{\prime}$ soit un point $P$ et la restriction de $f$ à $X-C$ donne un isomorphisme de $X-C$ sur $X^{\prime}-P$, autrement dit $C$ se contracte en un

Received November 8, 1971. 
point. On aurait donc $H^{0}\left(X^{\prime}-P, O_{X^{\prime}}\right)=H^{0}\left(X^{\prime}, O_{X^{\prime}}\right)=C^{1)}$. Puisque $X-$ $C$ est une variété de Stein, c'est une cotradiction.

\section{Condition Suffisante.}

2.1. En Géométrie Algébrique on a:

THÉORÈME (Goodman, Nakai). Soient $X$ une surface algébrique nonsingulière complète définie sur un corps algébriquement clos $k$ et $C$ une courbe irréductible complète sur $X$. Les conditions suivantes sont équivalentes:

(i) $X-C$ est affine.

(ii) Le diviseur $C$ est ample.

(iii) $(C \cdot Y)$ est positif pour toute courbe $Y$ sur $X$.

Pour la démonstration par exemple voir Hartshorne (5) Théorème 4.2 , p. 69 .

Soint $X$ une surface algébrique non-singulière complète définie sur $C$ et $C$ une courbe irréductible non-singulière sur $X$. Supposons que $X-C$ ne contient pas de courbes complètes. Alors d'apres le Théorème 1 et Théorème de Goodman-Nakai, on a :

$X-C$ est une variété de Stein si $\left(C^{2}\right)$ est positif et $X-C$ n'est pas une variété de Stein si $\left(C^{2}\right)$ est négatif.

On demande:

Problème A. $X-C$, est-elle une variété de Stein si $\left(C^{2}\right)$ est nul et $(C \cdot Y)$ est positif pour toute courbe $Y$ différente de $C$ ?

Remarque. Le problème est propre aux surfaces, algébriques, puisque si le degré de transcendance du corps des fonctions méromorphes sur $C$ est 1 , il n'existe pas de courbe sur $X$ satisfaisant à la condition du problème (voir Kodaira (7)).

2.2. Dans le cas où $X$ est une surface réglée et $C$ est une section, nous allons donner une condition suffisante pour que $X-C$ soit une variété de Stein.

1) dû à J. Frenkel et H. Matsumura (voir Narasimhan, R.: Introduction to the theory of analytic spaces, Lecture Notes in Mathematics, 25, Springer). Ils ont signalé plus généralement que $H^{0}\left(V-W, O_{V-W}\right)=H^{0}\left(V, O_{V}\right)=C$, si $V$ est un espace analytique compact et $W$ est un sous-ensemble analytique de codimension $\leq 2$. 
Soient $C$ une courbe complète non-singulière, $E$ une extension nontriviale du faisceau structural $O_{C}$ par $O_{C}$ déterminée par un élément nonnul $\xi$ de $H^{1}\left(C, O_{C}\right): 0 \rightarrow O_{C} \rightarrow E \rightarrow O_{C} \rightarrow 0$ (voir Atiyah (3)).

Désignons par $X$ le fibré projectif défini par $E$. On note, par abus de langage $C$ une section correspondant à la suite exacte ci-dessus. Singalons que $X-C$ est un espace fibré principal de groupe $\boldsymbol{G}_{a}$ et $O_{X}(1)$ est isomorphe à $O_{X}(C)$. Alors on a:

Lemme. $\quad\left(C^{2}\right)=0$.

Pour la démonstration voir Atiyah (3).

Proposition. $(C, Y)>0$ pour toute courbe $Y$ sur $X$ différente de $C$ si la caractéristique du corps de définition $k$ est nulle.

La démonstration est due à M. Maruyama. Puisque $X-C$ est un espace fibré principal de groupe $\boldsymbol{G}_{a}, \boldsymbol{G}_{a}$ opère sur $X$. Soit $Y$ une courbe sur $X . \quad Y$ est linéairement équivalente à $n C+\pi^{*} D$ où ( $D$ est un diviseur sur la base $C$ et $\pi$ est la projection de $X$ sur $C$ (voir Manin (8)). Singalons que, si l'on note $Y_{g}$ l'image de $Y$ par $g: X \rightarrow X g \in \boldsymbol{G}_{a}$, alors $Y$ est linéairement équivalent à $Y_{g}$. Nous allons démontrer $(C, Y)>0$ si $Y \neq C$. Si $Y$ est une section et $(C, Y)=0$, alors $Y$ est linéairement équivalent à $C+\pi^{*} D$ avec $\operatorname{deg} D=0$. Autrement dit $Y$ correspond à un sousfaisceau invertible de degré 0 de $E$ (voir Hartshorne (5), p. 51). Puisqu'il n'existe qu'un sous-faisceau invertible de degré 0 de $E$, on en conclut $Y=C$. Donc on peut supposer que $Y$ est irréductible et que $Y$ est ni une fibre ni une section. On a: $\left(Y^{2}\right)=\left(n C+\pi^{*} D \cdot n c+\pi^{*} D\right)=2\left(n c \cdot \pi^{*} D\right)$. Raisonnons par l'absurde. Si $(C \cdot Y)$ était nul, alors $0=(C \cdot Y)=\left(C \cdot \pi^{*} D\right)$ par conséquent $\left(Y^{2}\right)=0$. Soit $l$ une fibre générale et $l . Y=P_{1}+P_{2}+$ $\cdots+P_{r}$ avec $r \geq 2, P_{i} \neq P_{j}$ si $i \neq j$. Soit $g$ un élément de $G_{a}$ qui transforme $P_{1}$ à $P_{2}$. Puisque $Y_{g}$ est linéairement à $Y$ et $\left(Y^{2}\right)=0$, on aurait $Y_{g}=Y$. Donc $g$ induirait sur $P_{1}, P_{2}, \cdots, P_{r}$ une permutation. C'est une contradiction puisque l'opération de $\boldsymbol{G}_{a}$ sur $l=\boldsymbol{G}_{a}$ est l'addition et la caractéristique de $k$ est nulle.

2.3. A partir de maintenant nous supposons que toute variété algébrique est définie sur $C$. Soient $C$ une courbe algébrique non-singulière compléte, $\xi$ un èlèment de $H^{1}\left(C, O_{C}\right)$ et $L_{\xi}$ le sous-espace vectoriel de $H^{1}\left(C, O_{C}\right)$ engendré par $\xi$. La suite

$$
0 \longrightarrow Z \longrightarrow C \stackrel{\exp 2 \pi \sqrt{-1}()}{\longrightarrow} C^{*} \longrightarrow 0
$$


donne naissance à une suite exacte de cohomologie:

$$
\begin{aligned}
0 \longrightarrow H^{0}(C, Z) & \longrightarrow H^{0}\left(C, O_{C}\right) \longrightarrow H^{0}\left(C, O_{C}^{*}\right) \\
& \longrightarrow H^{1}(C, Z) \longrightarrow H^{1}\left(C, O_{C}\right) \longrightarrow H^{1}\left(C, O_{C}^{*}\right) .
\end{aligned}
$$

Puisque $H^{0}\left(C, O_{C}\right) \rightarrow H^{0}\left(C, O_{C}^{*}\right)$ est surjectif on a une suite exacte:

$$
0 \longrightarrow H^{1}(C, Z) \longrightarrow H^{1}\left(C, O_{C}\right) \longrightarrow H^{1}\left(C, O_{C}^{*}\right) \text {. }
$$

Signalons enfin que le groupe quotient $H^{1}\left(C, O_{C}\right) / H^{1}(C, Z)$ peut être identifié à la Jacobienne $J$ de $C$.

THÉORÈME 2. Si l'image de $L_{\xi}$ par l'application canonique de $H^{1}\left(C, O_{C}\right)$ dans $J$ est compacte et $\xi$ est non-nul, $X_{\xi}-C$ est une variété de Stein.

Puisque l'on déduit de la proposition que $X_{\xi}-C$ ne contient pas de sous-ensemble analytique compacte de dimension positive, d'après le théorème de Grauert, il suffit alors de vérifier que $X_{\xi}-C$ est holomorphe convexe. Par hypothése, il existe deux nombres $\lambda$ et $\mu$ tels que $\lambda \xi, \mu \xi$ forment une base d'un espace vetoriel réel $L_{\xi}$ et appartient à $H^{1}(C, Z)$. On peut supposer que les fonctions de transition de l'espace fibré principal $X_{\lambda \xi}-C$ de groupe $C$ et de base $C$ sont des nombres entiers. Soient maintenant $\left(U_{i}\right)$ un recouvrement ouvert de $C, Y$ un espace fibré principal de groupe $C$ et de base $C$ défini par $c_{i j} \in Z \subset\left(U_{i} \cap U_{j}, O_{C}\right)$ et $X_{i \xi}-C$ isomorphe à $Y$. Si l'on pose pour chaque trivialisation: $\tilde{e}_{i}: U_{i} \times \boldsymbol{C} \rightarrow \boldsymbol{C}$

$$
\left(x, z_{i}\right) \mapsto \exp 2 \pi \sqrt{-1} z_{i}
$$

(resp. $\tilde{e}_{i}: U_{i} \times C \rightarrow C$,

$$
\left.\left(x, z_{i}\right) \mapsto \exp -2 \pi \sqrt{-1} z_{i}\right)
$$

alors les $\tilde{e}_{i}$ (resp. $\tilde{e}_{i}$ ) se recollent et définissent une fonction holomorphe $\tilde{e}^{\lambda}$ (resp. $\left.\tilde{e}^{\lambda}\right)$ sur $Y$ et par isomorphisme une fonction holomorphe $e^{\lambda}\left(\operatorname{resp} . \bar{e}^{\lambda}\right)$ sur $X_{\lambda \xi}-C$. De la même manière on peut définir une fonction holomorphe $e^{\mu}$ (resp. $\bar{e}^{\mu}$ ) sur $X_{\mu \xi}-C$. Puisque $X_{\xi}-C$ est isomorphe (comme objet au-dessus de $C$ ) à $X_{\lambda \xi}-C$ (resp. $X_{\mu \xi}-C$ ) par conséquent on a quatre fonctions holomorphes $e^{\lambda}, \bar{e}^{\lambda}, e^{\mu}, \bar{e}^{\mu}$ sur $X_{\xi}-C$.

Signalons que, si $\left(w_{i}\right)_{i \in N}$ est une suite infinie de points de $C$ sans point adérent dans $C$, une des quatre fonctions $\exp \pm 2 \pi \lambda \sqrt{-1} w_{i}$, $\exp \pm 2 \pi \mu \sqrt{-1} w_{i}$ est non bornée, puisque $\lambda$ et $\mu$ sont linéairement indépendants sur $R$. Soit $\left(P_{i}\right)_{i \in N}$ une suite infinie de points de $X_{\xi}-C$ sans 
point adhérent dans $X_{\xi}-C$, en considérant que la base $C$ est compacte, on voit qu'une des quatre fonctions holomorphes sur $X_{\xi}-C e^{\lambda}\left(P_{i}\right), \bar{e}^{\lambda}\left(P_{i}\right)$, $e^{\mu}\left(P_{i}\right), \bar{e}^{\mu}\left(P_{i}\right)$ est non bornée d'où il résulte que $X_{\xi}-C$ est holomorphe convexe.

CoRollaire 1 (dû à Serre, voir Hartshorne (5), p. 232). Si le genre de $C$ est 1 et $\xi$ est un élément non-nul de $H^{1}\left(C, O_{C}\right), X^{\xi}$ est une variété de Stein.

En effect, les conditions du Théorème 2 sont vérifiées.

Corollaire 2. Si le genre de $C$ est 2 et la Jacobienne $J$ de $C$ est isomorphe à $E \times E$, ò̀ $E$ est une courbe elliptique, alors il existe une base $\omega_{0}, \omega_{1}$ de $H^{1}\left(C, O_{C}\right)$ ayant la propriété suivante: Soit $\xi=a_{0} \omega_{0}+a_{1} \omega_{1}$ est un élément non-nul de $H^{1}\left(C, O_{C}\right)$. Si le quotient $a_{1} / a_{0}$ est rationnel, $X_{\xi}-C$ est une variété de Stein.

Supposons que la courbe elliptique $E$ est définie par un sous-groupe discret de range 2 de $C$ engendré par $1, a$ où a n'appartient pas au corps des nombres réels $\boldsymbol{R}$. Puisque $J$ est isomorphe à $E \times E$ on peut identifier $J$ au groupe quotient $\boldsymbol{C} \oplus \boldsymbol{C} / \Gamma$ où $\Gamma$ est un sous-groupe discret de rang 4 de $C \oplus C$ engendré par $(1,0),(a, 0),(0,1),(0, a)$. Prenons $\omega_{0}=$ $(1,0), \omega_{1}=(0,1)$ comme base de $H^{1}\left(C, O_{C}\right)$. Si $\xi=a_{0} \omega_{0}+a_{1} \omega_{1}$ est une élément non-nul de $H^{1}\left(C, O_{C}\right)$ et le quotient $a_{1} / a_{0}$ est égal à $n / m$ avec $m$, $n \in Z$, alors $L_{\xi}$ contient deux points linéairement independants $\operatorname{sur} R(m, n)$ et $(m a, n a)$ de $\Gamma=H^{1}(C, Z)$. Autrement dit l'image de $L_{\xi}$ dans $J$ est compacte. D'après le Théorème $2 X_{\xi}-C$ est une variété de Stein.

Remarques 1. L'existence de courbes dont la Jacobienne soit isomorphe à $E \times E$ est assurée par Hayashida et Nishi (6).

2. Problème $\mathrm{A}^{\prime}$. Un espace fibré principal de groupe $\boldsymbol{C}$ dont la base est une surface de Riemann est holomorphe convexe?

Si le Problème A est affirmatif, le Problème $A^{\prime}$ l'est aussi. Inversment, $X_{\xi}-C$ est une variété de Stein pour $\xi \neq 0$, si le Problème $\mathrm{A}^{\prime}$ est affirmatif.

3. Si un espace fibré dont la base et la fibre sont des variétés de Stein est toujours une variété de Stein (voir Serre (9)), on peut démon- 
trer: $X_{\xi}-C$ est une variété de Stein si $L_{\xi}$ contient un élément non-nul de $H^{1}(C, Z)$.

\section{BIBLIOGRAPHIE}

[1] Andreotti, A. et Grauert, H., Théorème de finitude pour la cohomologie des espaces complexes, Bull. Soc. Math. France, 90 (1962), 193-259.

[2] Atiyah, M. F., Complex fibre bundles and ruled surfaces, Pro. London Math. Soc., (3) 5 (1955), 407-434.

[ 3 ] - - Complex analytic connections in fibre bundles, Trans. Amer. Math. Soc., 85 (1957), 181-207.

[4] Grauert, H., Über Modifikationen und exzeptionelle analytische Mengen, Nath. Ann., 146 (1962), 331-368.

[5] Hartshorne, R., Ample subvarieties of algebraic varieties, Lecture Notes in Mathematics, 156, Springer.

[6] Hayashida, T. and Nishi, M., Existence of curves of genus two on a product of two elliptic curves, Jour. of Math. Soc. of Japan, 17 (1965).

[ 7 ] Kodaira, K., On compact complex analytic surfaces, I, Ann. of Math., 71 (1960).

[8] Manin, Yu. I., Lectures on the K-functor in algebraic geometry, Russian Mathematical Surveys, 24 (1969).

[ 9 ] Serre, J.-P., Quelques problèmes globaux relatif aux variétés de Stein, Colloque sur les fonctions de plusieurs variables, C.B.R.M. Bruxelles, (1935), 57-68.

Nagoya University 